\title{
Performance Evaluation of Tunneling Mechanism in MIPv6 over IPv4
}

\author{
Ahmad-Ali KWIZERA ${ }^{1}$, Cemal KOÇAK ${ }^{2}$ \\ Computer Engineering, Gazi University Faculty of Technology, Ankara Turkey \\ 1wizados05@gmail.com, ${ }^{2}$ ccckocak@gazi.edu.tr \\ (Geliş/Received:10.03.2017; Kabul/Accepted:24.07.2017) \\ DOI: $10.17671 /$ gazibtd.331052
}

\begin{abstract}
MIPv6 protocol is designed to support the mobility of the nodes and maintain ongoing connections while changing their locations. Currently, most of the internet infrastructures are using IPv4. MIPv6 as an extension of IPv6 is not compatible with IPv4; in this case, the transition mechanisms were used to tunnel MIPv6 traffic through IPv4 internet. This paper analyzes the performance of Manual Tunneling and 6to4 Tunneling in MIPv6 Networks, which communicate across IPv4 internet. Applications like FTP, HTTP, Database and Email using performance parameters such as Throughput, Response Time and End-to-End Delay were compared to find the best transition mechanism for MIPv6 Networks. Simulations results show that Manual Tunneling performed better with 12\% than 6to4 Tunneling in Email, and HTTP applications with 13,54\%, whereas 6to4 Tunneling, outperforms Manual Tunneling in a Database application, while in FTP application, those two transition mechanisms have the same performance.
\end{abstract}

Keywords - MIPv6; Tunnel; Transition mechanisms; Manual Tunneling; 6to4 Tunneling

\section{MIPv6'daki Tünelleme Mekanizmasının IPv4 Üzerinden Performans Değerlendirmesi}

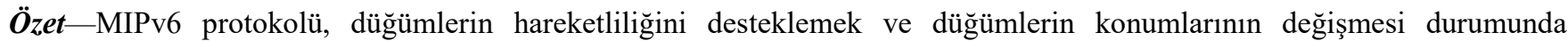
bağlantıların devamını sağlamak üzere tasarlanmıştır. Günümüz İnternet altyapısında çoğunlukla IPv4 kullanılmaktadır. IPv6'nın bir uzantısı olan MIPv6, IPv4 ile uyumlu olmadığından dolayı, IPv4 internet üzerinden MIPv6 trafiğinin tünelleme işlemi için geçiş mekanizmaları kullanılır. Bu makalede, IPv4 internet üzerinden iletişim kuran MIPv6 ağlarında Manuel tünelleme ve 6to4 tünelleme performansları analiz edilmiştir. FTP, HTTP, Veri tabanı ve Email gibi uygulamalar kullanılarak, MIPv6 ağlar için en iyi geçiş mekanizmasını bulmak için verimlilik (throughput), yanıt zamanı (response time) ve uçtan uça gecikme (end to end delay) gibi performans parametreleri karşılaştırılmıştır. Simülasyon sonuçlarına göre Manuel tünelleme Email uygulamasından \%12, HTTP uygulamasından ise \%13,54 daha iyidir. Manuel tünellemede veri tabanı uygulaması daha iyi performans gösterirken, FTP uygulaması 6to4 tünelleme mekanizması ve manuel tünelleme mekanizmasında aynı performansı göstermişlerdir.

Anahtar kelimler -MIPv6; Tünelleme; Geçiş Mekanizması; Manuel Tünelleme; 6to4 Tünelleme.

\section{INTRODUCTION}

After almost 30 years of IPv4 in use, the spectacular growth of wireless technology and fast internet are leading to the IPv4 addresses exhaustion. In order to meet the demands and overcome the problem of IP addresses depletion, many techniques were introduced such as Classless Inter-Domain Routing (CIDR) [1], Dynamic Host Configuration Protocol (DHCP) [2] and Network
Address Translation (NAT) [3].With the needs of modern networks, IPv4 address exhaustion was not only the problem but also supporting of new services, high mobility, Quality of Service (QoS) and high security were a problem [4].

A new IP (Internet Protocol Version 6-IPv6) was designed to solve IPv4 dissatisfactions with an extremely larger 
address space, which offer 128-bits addresses, excelling packet forwarding, smaller header size, high security, Multicast and Anycast Traffic support, QoS, Real Time Multimedia support, stateless autoconfiguration and better supporting mobility on the internet. Mobility support is a key feature, as everyone needs to be connected to the internet using mobile devices. Mobile IPv6 (MIPv6) supports node mobility on the internet with each mobile node identified by its home address, regardless of its current point of attachment. While away from its home IP subnet, a mobile node is also associated with Care-of Address (CoA), which indicates the mobile node's current location [5].

IPv6 specification was published twenty-three years ago in RFC 1883. Deployment of IPV6 is not going to be achieved overnight. It would be cumulative and step by step. IPv4 and IPv6 will coexist even after decades of IPv6 deployment [6] but the problem is that those two protocols are not compatible in communicating one with each other. In order to make the networks that use IPv4 and IPv6 to communicate with each other, various transition mechanisms have been developed and standardized to addresses specific transition and interoperability [7].

The incompatibility of IPv4 and IPv6 makes the evolution of current Internet to be built on transition mechanisms. The current transition mechanisms were primarily designed for fixed network. With the increase and popularity of mobile devices and wireless technology, some researchers are evaluating the performance and implementation in mobile network. Performance analysis of current transition mechanisms is necessary in order to develop improved transition mechanisms dedicated for mobile network and other types of modern networks.

IPv4 is still a dominant protocol. According to Google [8] up to December 26, 2016.23 years later, about $90.02 \%$ of the Internet is still using the overloaded IPv4 protocol. Therefore, tunneling mechanism was seemed to be the most suitable method to connect isolated IPv6 networks through IPv4 [9]. In this paper, we evaluate the performance of two Tunneling mechanisms: Manual Tunneling and 6to4 Tunneling in MIPv6 over IPv4. Their performances are compared in FTP, HTTP, Database and Email applications by measuring Throughput, Response Time and End-to-End Delay metrics. Our experiment is simulated in Riverbed Modeler. The rest of this paper is structured in the following manner: Section II discusses some of the related works done by other researchers. Section III presents a brief overview of the tunneling mechanisms studied in our experiment. Section IV describes the MIPv6 operation. In Section V, we provide our network architecture with tunneling mechanisms configurations in Riverbed Modeler and shows obtained performance results in the form of graphs and tables. Finally, the research is concluded in section VI.

\section{RELATED WORKS}

IPv6 transition mechanisms research area is not new in the literature. Early after the Internet Engineering Task Force (IETF) defines IPV6 protocol [10], researchers began to examine different transition mechanisms in order to bring a proper solution to tunnel IPv6 traffic into Internet of IPv4. However, there exist different transition mechanisms to allow IPv6 packet to be transmitted over IPv4 network and vice versa. Because of the dominant existence of IPv4 networks, the most studied transition mechanisms in the literature are IPv6 networks over IPv4 networks.

In [11], he studied the performance of MIPv6 over IPv6 network and using 6to4 transition mechanism. His network topology comprises of Correspondent Node (CN), which is a fixed node and a Mobile Node (MN). He evaluated performance metrics like throughput, traffic sent/received, and data dropped by MN in the pure IPv6 network and in the 6to4 network. His simulation results show that heavy applications perform better in all performance metric in pure IPv6 networks than in IPv4 network using 6to4 transition mechanism. He concludes that MIPv6 over pure IPv6 outperforms IPv4 using 6to4 transition mechanism, which is obvious because in IPv4 the packets had to be encapsulated.

In [12], they studied MIPv6 technology using Riverbed Modeler in the pure IPv6 internet. They analyzed application traffics while an MN is moving across Access Routers (ARs). They also look on the effect of MIPv6 routing mechanisms used by the $\mathrm{MN}$ in order to communicate with the $\mathrm{CN}$ on application response time, and impact of MIPv6 signaling in bidirectional tunneling and route optimization. They also evaluated the handover effects the congestion control in the transport layer. In their simulation scenario, the Correspondent nodes were not mobile. In their topology, the CNs were connected to the Central Routers (CRs), and Mobile Node was accessing routers that were present in different wireless networks.

In [13], the performance of configured Tunneling and 6to4Tunneling were evaluated on two different operating systems (Windows2008 and Windows 2012) using different performance parameters. Their experiment shows that 6to4 Tunneling outperformed configured Tunneling in more performance metrics, but their study was done in the pure fixed network. The study of [14] is similar to [13], the difference is that 6to4 Tunneling and configured tunnel were implemented on two different Linux distributions (Ubuntu and Fedora).The measured performance metrics results gave same values for the two transition mechanisms except for average delay which is different for those two tunnelings. This experiment was also driven in the fixed network. Although there have been many studies on IPv6 transition mechanisms, there are few 
types of research of transition mechanisms in the mobile network.

\section{IPv6 TUNNELING}

IPv6 Tunneling is a solution of sending IPv6 traffic or packet over IPv4 networks. A packet from the MIPv6 is encapsulated on sending node whenever it reaches a border router interface, which is connected to the IPv4 network. Then IPv4 infrastructure wraps and transports IPv6 packets as a payload [15]. A packet is decapsulated to the original when reaching to the destination node or destination border router interface. There are two categories of IPv6 Tunneling; Manual Tunneling and Automatic Tunneling. In our study, we use only Configured Tunneling and one of the automatic Tunnelings which is 6to4 Tunneling.

\subsection{Manual Tunneling}

The manual tunnel also called configured tunnel, static tunneling, protocol 41tunnels or 6in4 is employed to connect isolated IPv6 networks. This tunnel links permanently two IPv6 networks over an IPv4 backbone. At the tunnels source and destination devices, a tunnel sources and destinations are manually configured to build a static tunneling. The static routing table in the endpoints is also configured to determine packets to be tunneled [16].

\subsection{6to4 Tunneling}

6to4 Tunneling uses IPv4 infrastructure to allow remote IPv6 networks to communicate with each other. The IPv4 networks encapsulate IPv6 packets when a packet is transmitted between remote networks. A 6 to 4 address must have the following structure:

2002ipv4 address $>:<$ subnet $>\quad<$ interface identifier>

In order for it to allow communication between nodes, 6to4 mechanism requires a presence of relay routers [17].

\section{MOBILE IPv6 NETWORK OPERATIONS}

As described in RFC 3775, MIPv6 network is a network, which supports the movement of a single host from its point of attachment to another point of attachment on the internet. MIPv6 was developed to support node to be reachable and keeps ongoing connections during its movement to other locations within the topological requirement. The nodes that use MIPv6 are assigned two IPv6 addresses, Home Address (HoA) and a Care of Address (CoA). The HoA is assigned to nodes when they are at home subnets and is used for two reasons: mobile node, which is reachable with steady session through the communication, and the IP layer mobility, which is hidden from the upper layers. The advantage of keeping the HoA permanently for the mobile node is that all the
Correspondent nodes contact the mobile node through HoA without knowing the actual location of the mobile node, the packet will be forwarded to mobile node even if the mobile node is attached to a home subnet or not. If the mobile node is not attached to its home subnet, it is then the responsibility of the home agent to tunnel the packets to the mobile node's CoA [18].

The CoA is used when the mobile node is on the foreign network. When at the Foreign network a mobile node acquires a CoA based on the prefix of the foreign network. The CoA can be built with two mechanisms: stateful or stateless. Following the change of position and new address configuration, the mobile node must inform its home agent and the correspondent node (if routing optimization is used) of such changes by sending a Binding Update (BU) message. The $\mathrm{BU}$ is the configuration message in MIPv6 network that is encoded/ embedded in an optional extension header known as mobility header. The information contained in the BU is the HoA and the CoA of the mobile node. In order the HA and $\mathrm{CN}$ to forward packets directed to the home address of the mobile node, they need to store the BU from the mobile node. Once the mobile node, the HA, and $\mathrm{CN}$ have binding, all packets directed to the $\mathrm{MN}$ will be tunneled by $\mathrm{HA}$ and $\mathrm{CN}$ transmits all the packets directly to the $\mathrm{MN}$ if the route optimization is used [16].

\section{PERFORMANCE ANALYSIS}

In this study, Manual Tunneling and 6to4 Tunneling mechanisms are modeled to allow MIPv6 networks to communicate with each other through IPv4 Internet.

Applications like FTP, HTTP, Database and Email are used. In order to compare the performance of Manual Tunneling and 6to4 Tunneling in selected applications; Throughput, Response Time and End-to-End Delay as performance parameters are used.

\subsection{Network Architecture}

In this section, we show how our network is designed and manual tunneling and 6to4 tunneling are configured in Riverbed Modeler.

The Network model is composed of four Mobile IPv6 networks, which communicate over a native IPv4 network. This network model consists of two mobile nodes (MN1 and MN2) and four Access Routers that act as the Home Agent of MN1 (HA1), Home Agent of MN2 (HA2), Foreign Network of MN1 (FN1) and Foreign Network of MN2 (FN2). Each of these Agents consists of two interfaces: the wireless interface, which supports IEEE802.11b and connects the Mobile IPv6 Network and a wired interface, which connects these Agents to IPv4 Internet using serial line connection. The MN1 and MN2 are roaming from $\mathrm{HA} 1$ to $\mathrm{FN} 1$ and $\mathrm{HA} 2$ to $\mathrm{FN} 2$ 
respectively. All the Mobile IPv6 traffic between these two mobile nodes is tunneled through IPv4 Internet at the Agents IPv4 interface. The full depiction of the network model and topological parameters are shown in Fig.1 and Table 1.

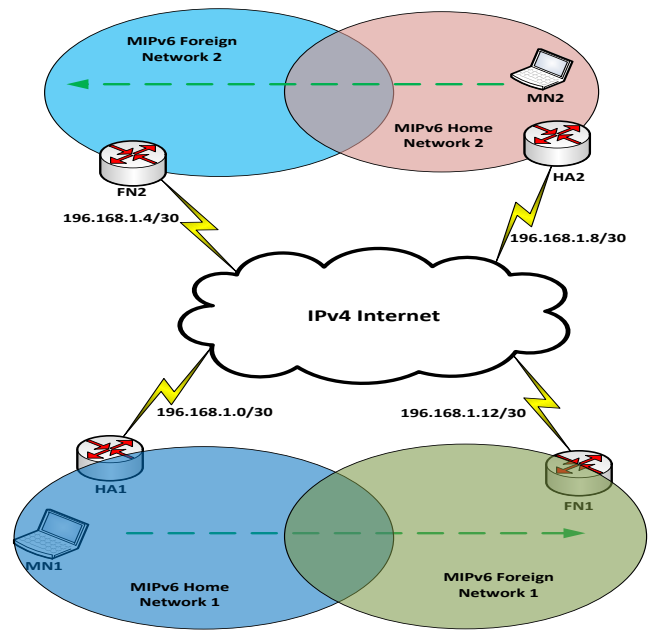

Fig. 1. Network model.

Table 1. Topological and network parameters

\subsubsection{6to4 Tunnel Configuration}

6 to 4 tunnel configuration requires that the IPv4 address used in the interface that connects to the internet be included in the tunnel address. Also, all the IPv6 addresses that are behind the router should include that IPv4 address and start by the prefix of 2002::/16. Table 2 shows the configuration of the 6to4 tunnel in the network.

Table 2. 6to4 tunnel configuration

\begin{tabular}{|c|c|}
\hline Name & Configuration \\
\hline Tunnel Mode & Tto4 \\
\hline Tunnel Interface & Tunnel0 \\
\hline \multirow{3}{*}{ Tunnel Addresses } & HA1=2002:196.168.1.2:624::624/64 \\
\cline { 2 - 2 } & FA1=2002:196.168.1.14:624::624/64 \\
\cline { 2 - 2 } & HA2=2002:196.168.1.10:624::624/64 \\
\cline { 2 - 2 } & FA2=2002:196.168.1.6:624::624/64 \\
\hline Static Routing & $2002:: 16$ \\
\hline Wireless Interfaces & HA1=2002: $196.168 .1 .2: \mathrm{A}:: 1 / 64$ \\
\cline { 2 - 2 } (MIPv6 networks) & FA1=2002:196.168.1.14:A::1/64 \\
\cline { 2 - 2 } & HA2=2002:196.168.1.10:A::1/64 \\
\cline { 2 - 2 } & FA2->2002:196.168.1.6:A::1 \\
\hline
\end{tabular}

\subsubsection{Manual Tunneling Configuration}

In order to set our Manual Tunneling on routers interfaces connected to IPv4 Internet, we must determine the tunnel mode, the tunnel source (IPv4 address), the tunnel destination (IPv4 address), and the tunnel's IPv6 address. In addition, to route traffic between IPv6 networks, we must specify static routes on our routers. Table 3 shows the configuration of the Manual tunnel in the network.
Table 3. Manual tunnel configuration

\begin{tabular}{|c|c|}
\hline Name & Configuration \\
\hline Tunnel Mode & 6to4 (Manual) \\
\hline Tunnel Interface & Tunnel0 \\
\hline \multirow{3}{*}{ Tunnel Addresses } & HA1-> 3ffe:b00:c18:1::1/112 \\
\cline { 2 - 2 } & FA1-> 3ffe:b00:c18:1::3/112 \\
\cline { 2 - 2 } & HA2-> 3ffe:b00:c18:1::2/112 \\
\cline { 2 - 2 } & FA2-> 3ffe:b00:c18:1::4/112 \\
\hline Static Routing & 3ffe:b00:c18:: /48 \\
\hline \multirow{3}{*}{$\begin{array}{c}\text { Wireless Interfaces (MIPv6 } \\
\text { networks) }\end{array}$} & HA1-> 3ffe:b00:c18:3:1/64 \\
\cline { 2 - 2 } & FA1-> 3ffe:b00:c18:4::1/64 \\
\cline { 2 - 2 } & HA2-> 3ffe:b00:c18:2::1/64 \\
\cline { 2 - 2 } & FA2-> 3ffe:b00:c18:5::1/64 \\
\hline
\end{tabular}

\subsection{Results Discussion}

This section presents and discusses the results obtained after the simulation of the previous network architecture in Riverbed Modeler. The performance metrics taken were Response Time, Throughput and End-to-End Delay. These performance parameters are explained below in this part to compare the two tunneling (Manual Tunneling and 6to4 Tunneling) performances in four applications (FTP, Database, HTTP and Email).

\begin{tabular}{|c|c|}
\hline Configuration & Parameters \\
\hline Topology_Size & $10 * 10 \mathrm{~km}$ \\
\hline Routers coverage area & $1248 \mathrm{~m}$ \\
\hline Simulation time & $15 \mathrm{~min}$ \\
\hline
\end{tabular}

\subsubsection{Response Time}

The response time represents the time elapsed between sending a request and receiving the response packet for the application. Fig. 2 to 5 show the performance results of response time metric of applications used in the simulation.

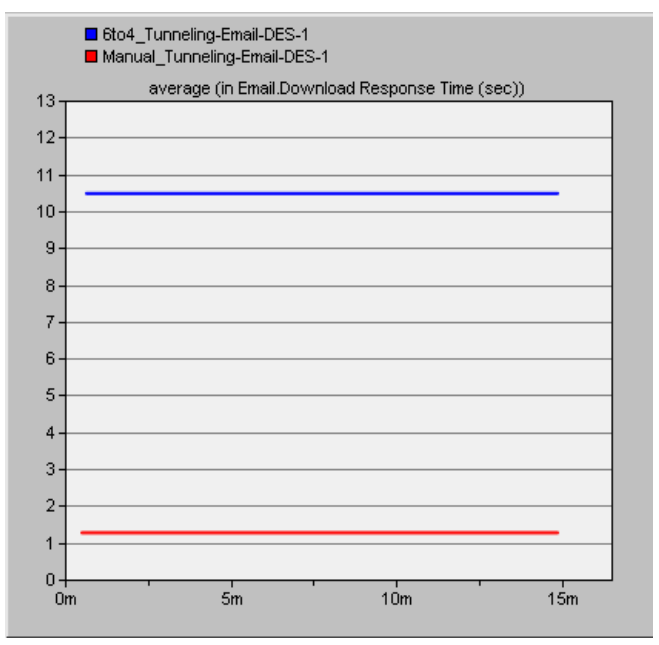

Fig. 2. Email Response Time. 


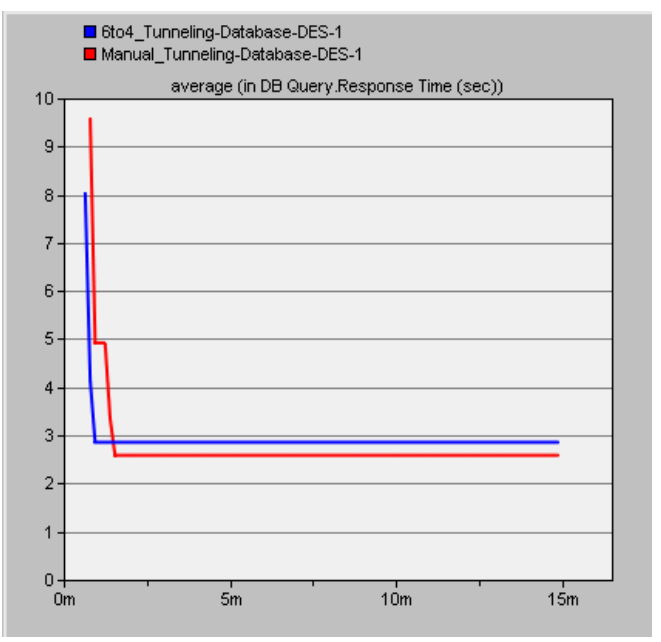

Fig. 3. Database Response Time.

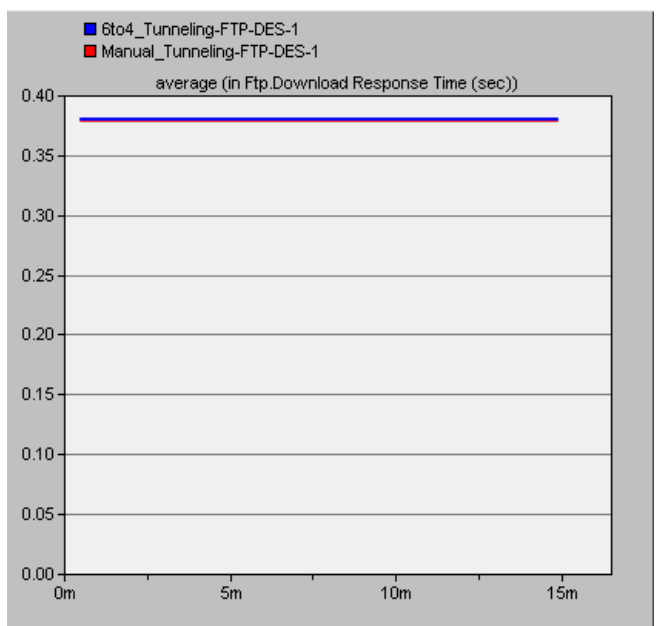

Fig. 4. FTP Response Time.

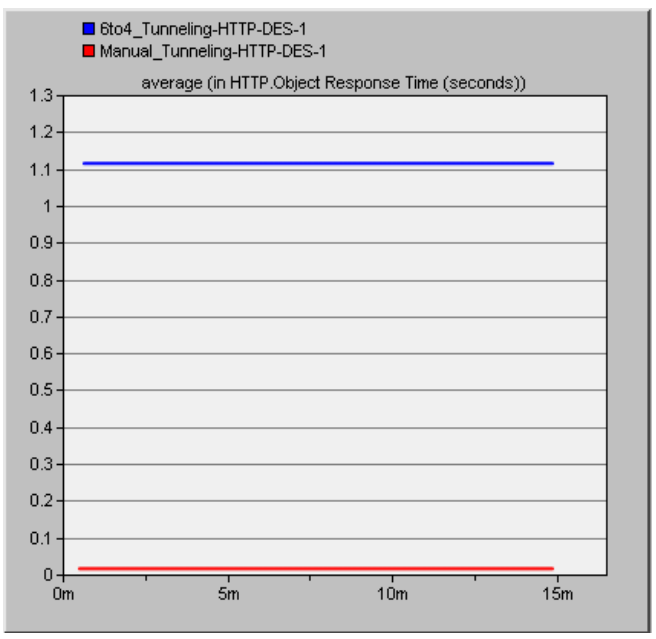

Fig.5. HTTP Response Time.

The Response Time for Email in Manual Tunneling is $1.2687 \mathrm{sec}$ and $10.4765 \mathrm{sec}$ in 6 to 4 Tunneling as shown in Fig. 2. This shows that the Manual Tunneling response time is approximately 8 times faster than the response time of 6to4 Tunneling. In the case of a Database application, at the beginning, 6to4 Tunneling had smaller response time than the response time of Manual Tunneling, but after the first minute, Manual Tunneling response time began to have a better performance until the end of simulation as depicted in Fig. 3. The Response Time of FTP application in Manual Tunneling and 6to4 Tunneling is nearly equal (0.3791sec for Manual Tunneling and 0.38 for 6to4 Tunneling) as shown in Fig.4. Fig.5 shows the Response Time of Manual Tunneling and 6to4 Tunneling mechanisms in HTTP application as 0.0155 and 1.1144 respectively. It means that 6to4 Tunneling response time is 71 times slower than Manual Tunneling response time.

In general, we observed that manual tunneling has less response time than 6to4 tunneling, which means that it has a better performance. This is summarized clearly in Table 4 below.

Table 4. Summary of Response Time in Manual and 6to4 Tunneling

\begin{tabular}{|c|c|c|}
\hline \multirow{2}{*}{ Applications } & \multicolumn{2}{|c|}{ Response Time (sec) } \\
\cline { 2 - 3 } & $\begin{array}{c}\text { Manual } \\
\text { tunneling }\end{array}$ & $\begin{array}{c}\text { 6to4 } \\
\text { tunneling }\end{array}$ \\
\hline E-mail & 1.2687 & 10.4765 \\
\hline Database & 2.587 & 2.856 \\
\hline FTP & 0.3791 & 0.38 \\
\hline HTTP & 0.0155 & 0.1144 \\
\hline
\end{tabular}

\subsubsection{Throughput}

It is the average of data transferred across a medium per unit time. A higher throughput means a better performance for the network. The following throughput results were taken at link HA1 from our experiment. Fig. 6 to 9 show the performance results of throughput metric of applications used in the simulation.

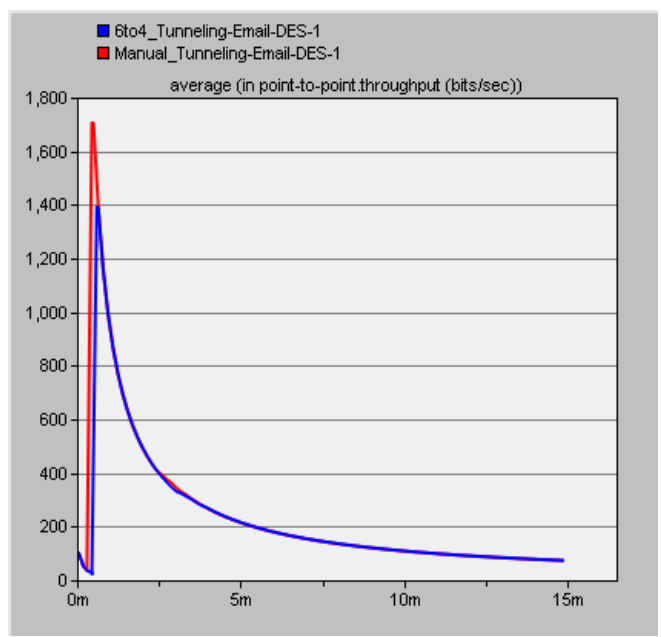

Fig. 6. Email Throughput. 


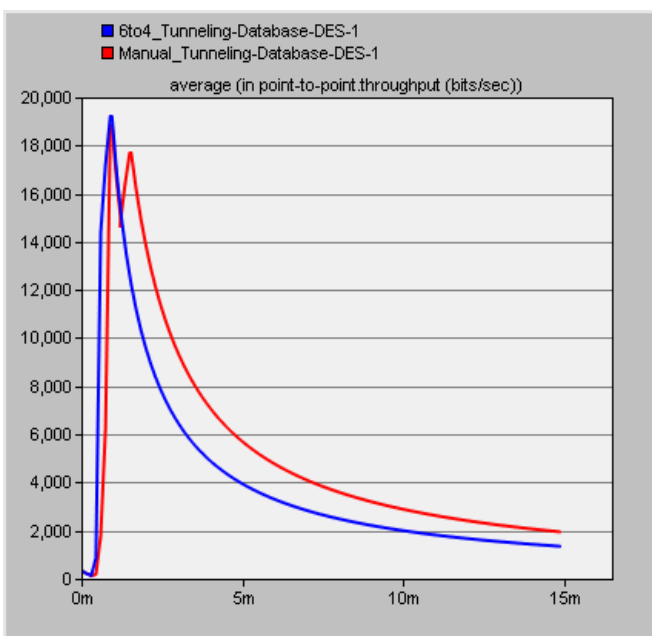

Fig. 7. Database Throughput.

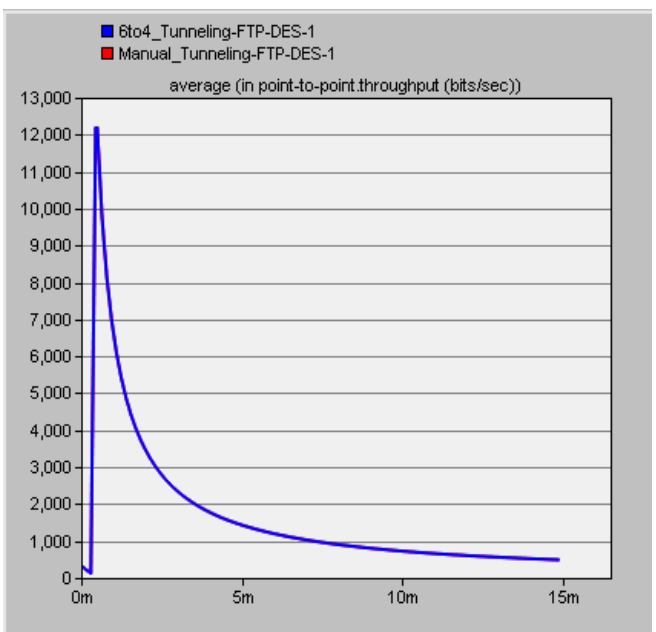

Fig. 8. FTP Throughput.

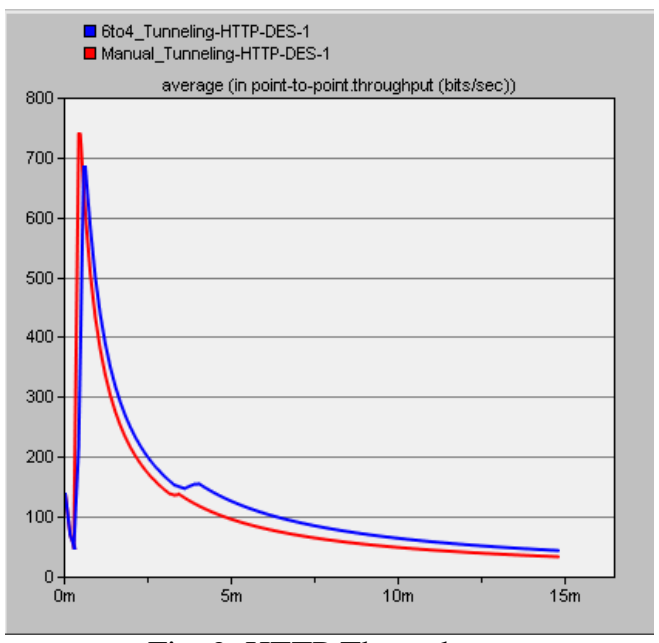

Fig. 9. HTTP Throughput.

As seen in an Email application, manual tunneling has higher throughput when compared to 6to4tunneling, their respective peak values are $214 \mathrm{bytes} / \mathrm{sec}$ and $174 \mathrm{bytes} / \mathrm{sec}$ as shown in Fig. 6. In a database application, the 6to4 mechanism has a greater throughput than manual tunneling as seen in Fig. 7 above with values of 2410 bytes/sec and 2360 bytes/sec respectively. But after the first 2 minutes, the performances changed and the simulation ends when Manual Tunneling has 244 bytes/sec as final value while 6to4 Tunneling has 169 bytes/sec as the final value. Fig. 8, shows that Manual Tunneling has the same Throughput result as 6to4 Tunneling with $1529 \mathrm{bytes} / \mathrm{sec}$, as maximal value and at the end with 61 bytes/sec, as a final value in FTP application. For a web application, the throughput results between Manual Tunneling and 6to4 Tunneling are closer; with the respective values of $93 \mathrm{bytes} / \mathrm{sec}$ and $86 \mathrm{bytes} / \mathrm{sec}$ as shown in Fig. 9.The Manual Tunneling Throughput is higher than the 6to4 Tunneling one.

In general, Manual Tunneling performed slightly better than 6to4 Tunneling in Email and HTTP applications, in contrast, 6to4 Tunneling has slightly better throughput when compared to manual tunneling in a Database application, while in FTP application, Manual Tunneling and 6to4 Tunneling, have the same performance. The throughput results of FTP, Email, HTTP and Database applications in Manual and 6to4 Tunneling Mechanisms are summarized in Table 5 below.

Table 5. Summary of Throughput in Manual and 6to4 Tunneling

\begin{tabular}{|c|c|c|}
\hline \multirow{2}{*}{ Applications } & \multicolumn{2}{|c|}{ Throughput (bytes/sec) } \\
\cline { 2 - 3 } & $\begin{array}{c}\text { Manual } \\
\text { tunneling }\end{array}$ & 6to4 tunneling \\
\hline E-mail & 214 & 174 \\
\hline Database & 244 & 169 \\
\hline FTP & 61 & 61 \\
\hline HTTP & 93 & 86 \\
\hline
\end{tabular}

\subsubsection{End-to-End Delay}

It is the difference between the time a packet arrives at its destination and the creation time of the packet. In our study, End-to-End Delay parameter was taken at mobile node (MN). Fig. 10 to 13 show the performance results of End-to-End Delay metric of applications used in the simulation. 


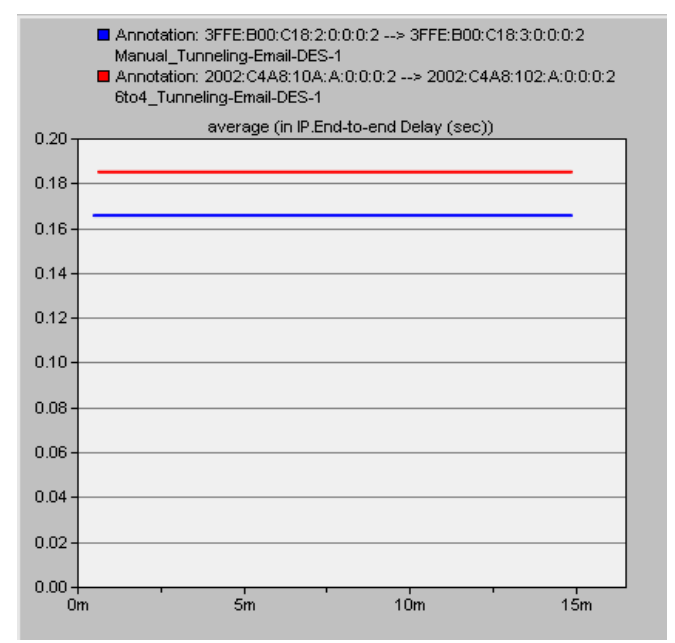

Fig.10. Email End-to-End Delay.

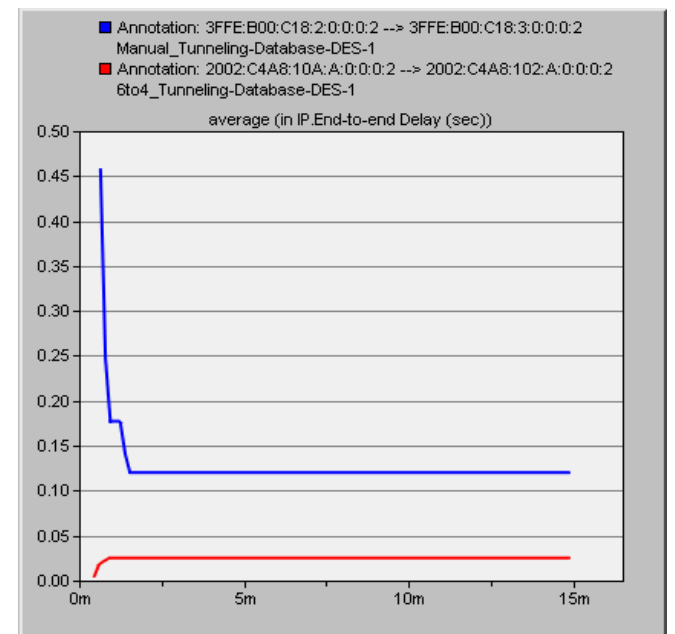

Fig.11. Database End-to-End Delay.

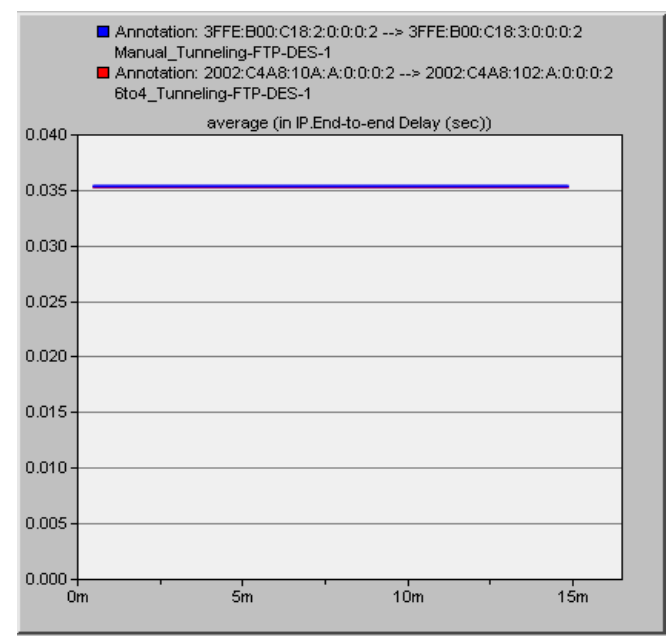

Fig.12. FTP End-to-End Delay.

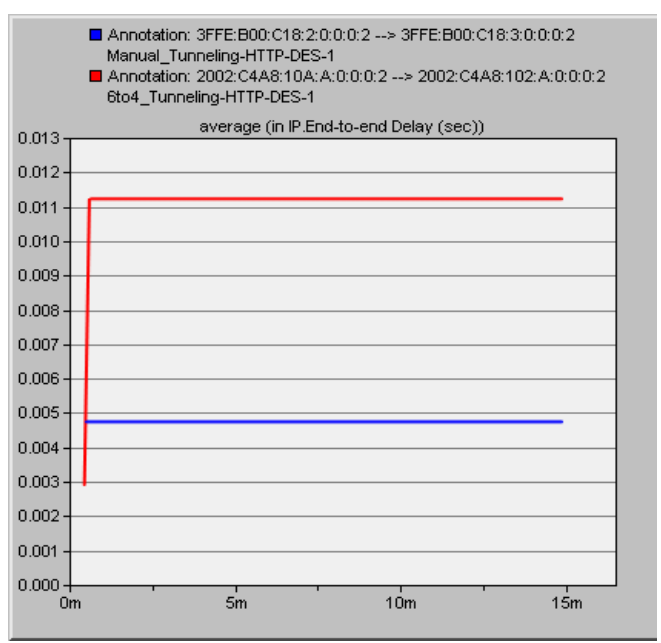

Fig.13. HTTP End-to-End Delay.

As shown in Fig.10, End-to-End delay of E-mail application using 6to4 tunneling mechanism is $0.1849 \mathrm{sec}$ and for Manual tunneling mechanism is $0.1656 \mathrm{sec}$. This means that Manual Tunneling performed better than 6to4 Tunneling in the Email application. In Fig. 11, the End-toEnd delay value of Manual Tunneling in Database application after 90 seconds of simulation time dropped to $0.1201 \mathrm{sec}$ while the End-to-End delay value of 6to4 Tunneling jumped to $0.0045 \mathrm{sec}$. Consequently, this shows that 6to4 Tunneling outperforms Manual Tunneling in a Database application. For FTP application, the Endto-End Delay metric values using both transition mechanisms (6to4 and Manual Tunneling) are the same with $0.0353 \mathrm{sec}$. Fig. 12 shows that those two mechanisms performed homogeneously. It is too clear in Fig.13 that depicts HTTP application, at the beginning of the simulation; Manual Tunneling had a greater value than 6 to4 Tunneling. But after $70 \mathrm{sec}$ of simulation time, the value of 6 to 4 Tunneling increased whereas the Manual Tunneling remained the same until the end of the simulation, which makes Manual Tunneling to have better performance than 6to4 Tunneling with values of 0.0047 $\mathrm{sec}$ and $0.0112 \mathrm{sec}$ respectively. The End-to-End Delay results are summarized in Table 6 below.

Table 6. Summary of End-to-End Delay in Manual and 6to4 Tunneling

\begin{tabular}{|c|c|c|}
\hline \multirow{2}{*}{ Applications } & \multicolumn{2}{|c|}{ End-to-End Delay (sec) } \\
\cline { 2 - 3 } & $\begin{array}{c}\text { Manual } \\
\text { tunneling }\end{array}$ & 6to4 tunneling \\
\hline E-mail & 0.1656 & 0.1849 \\
\hline Database & 0.1201 & 0.0045 \\
\hline FTP & 0.035 & 0.035 \\
\hline HTTP & 0.0047 & 0.0112 \\
\hline
\end{tabular}




\section{CONCLUSION}

In this paper, we evaluated the performance of Manual Tunneling and 6to4 Tunneling mechanism by measuring different performance metrics (Response Time, Throughput, and End-to-End Delay) of different applications (Email, Database, FTP, and HTTP) in MIPv6 where separate MIPv6 networks are connected via IPv4 Internet. In examining all the results obtained from Riverbed Modeler, Manual Tunneling is shown to have better than 6to4 Tunneling in Email and HTTP applications in all performance parameters except in Database application where it is contrary, while in FTP application both two transition mechanisms have the same performance level in all parameters. Even if Manual Tunnel performances were shown to have outperformed 6to4 Tunneling in many applications, the manual configuration makes it more complex and difficulty than 6to4 Tunneling which is automatic. Therefore, Manual Tunneling is more suitable for small networks. However, Manual Tunneling performance can be predicted when compared with 6to4Tunneling because there is a fixed and single remote tunnel end point.

\section{REFERENCES}

[1] V. Fuller, T. Li, J. Yu, and K. Varadhan, "Classless Inter-Domain Routing (CIDR): an Address Assignment and Aggregation Strategy," RFC 1519, September 1993.

[2] R. Droms, J. Bound, B. Volz, C. Perkins, T. Lemon, and M. Carney, "Dynamic Host Configuration Protocol for IPv6 (DHCPv6)," RFC 3315, July 2003.

[3] C. Huitema, "Teredo: Tunneling IPv6 over UDP through network address translations (NATs)," RFC 4380, February 2006.

[4] S. Narayan and S. Tauch, "IPv4-v6 Transition Mechanisms Network Performance Evaluation on Operating Systems," Computer Science and Information Technology (ICCSIT), 3rd IEEE International Conference on, 664-668. IEEE, July 2010.

[5] F. A. T. Al-Saedi, and M. M. Asem, "Performance Study of Mobile IPv6 Using OPNET," In International Journal of Engineering Research and Technology, 3(8),549-557 2014.

[6] R. Gilligan and E. Nordmar, "Transition Mechanisms for IPv6 Hosts and Routers,”. RFC1933. April 1996.
[7] N. Bahaman, E. Hamid and A. S. Prabuwono, "Network performance evaluation of 6to4 tunneling," In Innovation Management and Technology Research (ICIMTR), International Conference on, 263-268. IEEE, 2012.

[8] Internet: V. B. Iljitsch, "IPv6 celebrates its 20th birthday by reaching 10 percent deployment," March 2016, Available: http://arstechnica.com/business/2016/01/ipv6-celebrates-its-20thbirthday-by- reaching-10-percent-deployment/

[9] S. Kalwar, N. Bohra, and A. A. Memon, "A survey of transition mechanisms from IPv4 to IPv6-Simulated test bed and analysis," in Digital Information, Networking, and Wireless Communications (DINWC), 2015 Third International Conference on, 30-34, 2015.

[10] S. Deering and R.Hinden 'Internet Protocol Version 6 (IPV6) Specification,”RFC 1883, December 1995.

[11] A.O. Alwer, "Performance Evaluation for MIPv6 in Pure IPv6 Networks vs. 6to4 IP Mechanism Networks using OPNET," International Journal Of Electronics, Mechanical And Mechatronics Engineering, 1317-1326, 2016.

[12] D.Le, X.Fu, and D.Hogrefe, " Evaluation of Mobile IPv6 Based on an OPNET Model," In in Proceedings of The 8th International Conference for Young Computer Scientists, 1-7, 2005.

[13] D.Hadiya, R.Save, and G.Geetu, "Network performance evaluation of 6to4 and configured tunnel transition mechanisms: An empirical test-bed analysis, "6th International Conference on Emerging Trends in Engineering and Technology, 56-60, 2013.

[14] S. Narayan and S. Tauch, "IPv4-v6 configured tunnel and 6to4 transition mechanisms network performance evaluation on Linux operating systems," In Signal Processing Systems (ICSPS), 2nd International Conference on, 113-117. IEEE, 2010.

[15] R. E. Gilligan and E. Nordmark"Transition mechanisms for IPv6 hosts and routers," RFC 2893, August 2000.

[16] J. Hagino and K. Yamamoto, "An IPv6-to-IPv4 Transport Relay Translator," RFC 3142, June 2001.

[17] Y. Sookun, and V. Bassoo, "Performance analysis of IPv4/IPv6 transition techniques," In Emerging Technologies and Innovative Business Practices for the Transformation of Societies (EmergiTech), IEEE International Conference on, 188-193. DOI: 10.1109/EmergiTech.2016.7737336, 2016.

[18] D. Johnson, C. Perkins, and J. Arkko, "Mobility support in IPv6," RFC 3775, June 2004. 